\title{
Investigación y desarrollo de un nuevo producto para emprendimiento de microeconomía popular y solidaria.
}

Research and development of a new product for a popular and solidarity microeconomic enterprise.

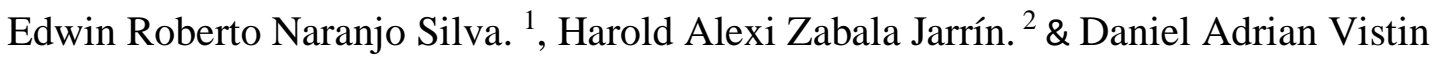
Guamantaqui. ${ }^{3}$

\begin{abstract}
.
DOI: https://doi.org/10.33262/concienciadigital.v4i3.1.1819

Introduction. This investigative document focused on developing a new service for the Food Service Association "ASO KARAKUNA", a member of the Popular and Solidarity Economy actors located in the Veloz parish, Riobamba canton, Chimborazo province. Objective. Create a significant differentiation process in contrast to its competitors in the reference market, likewise the new product will increase the recognition of the corporate image and the optimization of its resources. Methodology. Starting from a process of weighing ideas through matrices through the method of experts, in the same way we worked with the association, proposing product alternatives, such as catering for banquets and buffet for social events, with the support of research techniques from markets such as the survey through the application of the instrument known as the questionnaire to

${ }^{1}$ Escuela Superior Politécnica de Chimborazo, Facultad de Recursos Naturales, Carrera de Turismo, Riobamba, Ecuador, edwin.naranjo@espoch.edu.ec, https://orcid.org/0000-0002-2067-1085

2 Escuela Superior Politécnica de Chimborazo. Facultad de Administración de Empresas Carrera de Ingeniería en Marketing, Riobamba, zabalaj28@ gmail.com, https://orcid.org/0000-0003-1213-0729

${ }^{3}$ Escuela Superior Politécnica de Chimborazo. Facultad de Recursos Naturales. Carrera de Agronomía, Orellana, Ecuador, daniel.vistin@espoch.edu.ec, https://orcid.org/0000-0002-8313-9176
\end{abstract}


measure the degree of acceptance of the products and the consumer profile, furthermore, the company's brand manual was developed together with the strategies for the association to position itself in the minds of consumers. Results. As a general result of the variables measured, it was determined that services will be offered according to the generalized economy of the standard population that comprises between $\$ 350$ to $\$ 500$ US dollars, in the same way the proposed products have been classified into three ranges according to the Omnes method, with a price coefficient slightly equal to the market minimum for each of the products. Conclution. It was concluded that the demand for these services is requested by people in an age range between 18 to 35 years old, in terms of gender they are similar, with men and women reporting 55\% and $45 \%$ respectively according to the tabulation of the surveys.

Keywords: Product Research and Development, Product, Marketing, Popular solidarity economy.

\section{Resumen.}

Introducción. El presente documento investigativo se enfocó en desarrollar un nuevo servicio para la Asociación de servicios de alimentos "ASO KARAKUNA" miembro de los actores de la Economía Popular y Solidaria ubicada en la parroquia Veloz, cantón Riobamba, provincia de Chimborazo. Objetivo. Crear un proceso de diferenciación significativa en contraste con sus competidores en el mercado de referencia, asimismo el producto nuevo incrementara el reconocimiento de la imagen corporativa y la optimización la de sus recursos. Metodología. Partiendo desde un proceso de ponderación de ideas mediante matrices a través del método de expertos, del mismo modo se trabajó con la asociación, planteando alternativas de productos, como el catering para banquetes y buffet para eventos sociales, con la sustentación de técnicas de investigación de mercados como la encuesta mediante la aplicación del instrumento conocido como el cuestionario para medir el grado de aceptación de los productos y el perfil del consumidor, más aún se elaboró el manual de marca de la empresa junto con las estrategias para que la asociación se posicione en la mente de los consumidores. Resultados. Como resultado general de las variables medidas se determinó que se ofertará servicios de acuerdo a la economía generalizada de la población estándar que comprende entre $\$ 350$ a $\$ 500$ dólares americanos, de igual manera los productos propuestos se los ha clasificado en tres gamas según el método de Omnes, con un coeficiente de precio ligeramente igual al mínimo de mercado para cada uno de los productos. Conclusión. Se concluyo que la demanda de estos servicios es solicitada por las personas en un rango de edad de entre 18 a 35 años, en cuanto al género son similares reportándose hombres y mujeres con un $55 \%$ y $45 \%$ respectivamente de acuerdo a la tabulación de las encuestas.

Palabras Clave: Investigación y desarrollo de Producto, Producto, Marketing, Economía popular solidaria. 


\section{Introducción.}

En Ecuador a partir de la nueva constitución del 2008, se reconoce a la economía popular y solidaria EPS como el cuarto sector de la economía nacional. Según el art. 283 manifiesta, "El sistema económico es social y solidario; reconoce al ser humano como sujeto y fin; propende a una relación dinámica y equilibrada entre sociedad, Estado y mercado, en armonía con la naturaleza; y tiene por objetivo garantizar la producción y reproducción de las condiciones materiales e inmateriales que posibiliten el buen vivir. El sistema económico se integrará por las formas de organización económica pública, privada, mixta, popular y solidaria, y las demás que la Constitución determine. La economía popular y solidaria se regulará de acuerdo con la ley e incluirá a los sectores cooperativistas, asociativos y comunitarios (EPS, 2011).

Alrededor de cuatro millones de ecuatorianos buscan en la EPS una alternativa para salir de los niveles de pobreza en los que se mantienen. Para apoyar este objetivo el estado creó el Instituto Nacional de Economía Popular y Solidaria (IEPS), como entidad de derecho público, adscrito al Ministerio de Inclusión Económica y Social (MIES), con jurisdicción nacional, dotado de personalidad jurídica, patrimonio propio e independencia técnica, administrativa y financiera." Según el Decreto Ejecutivo No. 1668, 2009 (SEMPLADES, 2013)

Los actores involucrados en el círculo de la EPS en el Ecuador aportan desde sus plataformas con bases y experiencias para la consolidación de este marco socioeconómico y la mejora continua de su calidad de vida. En este contexto, la presente investigación busca desarrollar un nuevo servicio para la Asociación de servicios de alimentos "Aso kara kuna" miembro de los actores empresariales de la EPS, realizando el estudio de diagnóstico interno y externo de la asociación para determinar su situación actual, de este modo analizara e interpretara los datos obtenidos para determinar de este modo el perfil del consumidor y el grado de aceptación del nuevo producto, plasmando estrategias que permitan el crecimiento de la asociación.

El marketing, dentro de los emprendimientos es mucho más que simples actividades enfocadas en las ventas, más bien es una actitud que busca que una empresa tenga innovación, que conozca sus riesgos en todas las decisiones que tome, con el fin de cumplir sus objetivos empresariales (Sánchez y Joaquín, 2010). Por otro parte es una manera sutil de comprender los negocios (Gómez et al., 2012), es una forma positiva de realizar el trabajo, es por ende la esencia de toda organización, que va de la mano con la satisfacción de todos sus clientes (Pérez y David, 2018).

Asimismo, el marketing se centra especialmente en el cliente para llegar a cumplir los objetivos de la empresa (Lambin y Jacques, 1995), y así poder combatir a la competencia, creando valor agregado a sus clientes potenciales y reales (Kotler y Keller, 2016). El producto es uno de los elementos de mayor importancia para el marketing, el mismo que se puede definir como un servicio o artículo que se pueda ofrecer en el mercado para su atención, adquisición, uso o consumo, para satisfacer un deseo o una necesidad (Mesa, 2012). De la misma forma es un elemento tangible por medio del cual la empresa satisface 
las necesidades (Garnica y Maubert, 2009), y deseos de los consumidores y en consecuencia genera ganancias y rentabilidad para la organización (Canales, 2013).

En síntesis, el marketing es una ciencia administrativa que las organizaciones utilizan con la finalidad de atraer y retener al cliente (Gravens y Piercy, 2007), satisfaciendo sus deseos y necesidades mediante los bienes y servicios que ofrece las mismas a cambio de rentabilidad y del posicionamiento en el mercado (Vallet, 2015).

La investigación y desarrollo del producto, da respuesta a las necesidades variables del mercado con la finalidad de liderar el mercado mediante la introducción de nuevos artículos y servicios al mercado con la mejora de los productos existentes (Hoffman et al., 2007), si la empresa no tiene la capacidad monetaria para emprender un proceso de investigación y desarrollo puede crear un proceso de mejora involucrando a la mayor cantidad de sus colaboradores (Liton, 2019).

La Economía Popular y Solidaria, se concibió como la forma de organizar económicamente a cada uno de sus integrantes de forma particular o colectiva, además participan activamente en procesos de producción, comercialización, intercambio, financiamiento y consumo de bienes y servicios de tal manera que sean capaces de satisfacer necesidades y generar ingresos mejorando la estabilidad economía de cada una de las personas que forman parte de la actividad económica y productiva (Zabala et al., 2017).

El planteamiento de la economía popular y solidaria EPS se conformó de tal manera que la solidaridad, cooperación y reciprocidad sean los ejes principales para mejorar el trabajo y al ser humano como sujeto y fin de su actividad, orientada al buen vivir, en armonía y en naturaleza aunque el lucro y la acumulación del capital es indispensable para mantener un buen nivel de vida, los objetivos de la EPS van más allá de solo el aspecto económico porque busca generar beneficio comunitario (Pacheco, 2018).

Por esta razón se plantea seguir con la búsqueda del buen vivir y del bien común, la prelación del trabajo sobre el capital y de los intereses colectivos sobre los individuales, el comercio justo y consumo ético y responsable, la equidad de género, el respeto a la identidad cultural, la autogestión, la responsabilidad social y ambiental, la solidaridad y rendición de cuentas y la distribución equitativa y solidaria de excedentes (Pacheco, 2018).

\section{Metodologia.}

Este documento contiene concepto claves sobre la mezcla de marketing, que permite tener una referencia de cómo se va a establecer el producto, además se debe tomar en cuenta las variables que conforman el producto como son sus características, diseño, marca, precio y funcionalidad, que son fundamentales para desarrollarlo, siendo importante considerar que mediante su elaboración contribuirá al enriquecimiento de conocimientos.

El presente estudio es de nivel descriptivo debido a que busca familiarizarse con el problema del estudio, además de determinar cuáles son las causas primordiales que 
incitan al problema de investigación complementado mediante la investigación bibliográfica que se basa en la recopilación de aportes de diferentes autores sobre aspectos relacionados al desarrollo del producto, dichas definiciones dotan un mejor entendimiento de la realidad del marketing dentro del mercado local. Se realizó lluvia de ideas para describir los productos innovadores, complementando el mismo con el método de expertos y una regla de decisión se puedo seleccionar los productos más óptimos.

Se generó la lluvia de ideas entre las personas que laboran en la asociación y el grupo de trabajo, lo cual permitió elegir las ideas más favorables, luego llevándose a cabo a mediante una investigación de mercados, utilizando un enfoque cuantitativo, la técnica utilizada fue la encuesta , con un cuestionario estructurado no disfrazado, ayudando a la recolección, registro y análisis de datos representativos de una población, la misma que fue aplicada en las parroquias urbanas de la ciudad de Riobamba, teniendo en Lizarzaburu el 33\%, Maldonado el 22\%, Velazco el $24 \%$,Veloz el 15\% con y finalmente Yaruquíes con $6 \%$, para medir la factibilidad del producto en el mercado ,considerando los resultados obtenidos se puede definir que los productos deben enfocarse en las necesidades de los consumidores y cumplir con todas sus expectativas, generando una promesa de valor, puesto que la llamada sociedad de consumo se enfoca en la innovación y perfeccionamiento del producto.

Se ejecutó la investigación de mercados en la zona urbana de Riobamba, a través de la aplicación de cuestionarios estructurados no disfrazados, haciendo uso de escalas de Likert, en primera instancia con la aplicación de encuestas piloto y posteriormente las encuestas finales en función de las siguientes variables, Frecuencia de asistencia, Solicitud del servicio, Variedad de productos, Importancia del servicio, Tiempos de entrega, Decisión de compra, Tipo de servicio, Preferencias de catering y Aceptación del servicio.

Para determinar la cantidad de encuestas a aplicar se utilizó la fórmula para calcular la muestra cuando se conoce el tamaño de la población, sin embargo, se complementó con la proyección poblacional para el año 2019:

Fórmula para la base poblacional para realizar la proyección del 2019.

$$
\begin{aligned}
& \text { Fórmula } \\
& \qquad n=\frac{N \cdot Z^{2} \cdot p \cdot q}{e^{2}(N-1)+Z^{2} \cdot p \cdot q}
\end{aligned}
$$

\section{Donde:}

$\mathrm{N}=$ Tamaño de la población

$\mathrm{P}=$ Probabilidad de que el evento ocurra.

$\mathrm{Q}=$ Probabilidad de que el evento no ocurra.

$\mathrm{e}=$ Error de estimación o error maestral

$\mathrm{Z}=$ Margen de confiabilidad

$$
\begin{aligned}
& \mathbf{N}=\mathbf{8 3 3 7 4} \\
& P=0,80 \\
& Q=0,20 \\
& e=0,05(5 \%) \\
& Z=1,96
\end{aligned}
$$

$$
n=\frac{51246,29}{209,04} \quad n=246
$$


La cantidad de personas a encuestar es de $n=246$, por esta razón se aplicó el proceso de estratificación en tomando en cuenta la población por cada una de las parroquias urbanas y el porcentaje que representan, de tal manera que la muestra obtenida es representativa.

Tabla 1. Estratificación de las encuestas

\begin{tabular}{cccc}
\hline Parroquia & Población & Porcentaje & Muestra \\
\hline Lizarzaburu & 27301 & $33 \%$ & 81 \\
Maldonado & 18309 & $22 \%$ & 54 \\
Velazco & 19944 & $24 \%$ & 59 \\
Veloz & 12588 & $15 \%$ & 37 \\
Yaruquíes & 5232 & $6 \%$ & 15 \\
TOTAL & $\mathbf{8 3 3 7 4}$ & $\mathbf{1 0 0 \%}$ & $\mathbf{2 4 6}$ \\
\hline
\end{tabular}

Fuente: Elaboración propia

\section{Resultados.}

Entre los principales resultados generados de las variables de estudio, se pudo determinar que en relación al género, los porcentajes de participación en el estudio son muy similares con $55 \%$ y $45 \%$ de hombres y mujeres respectivamente, de igual manera más de la mitad de los encuestados tienen un nivel de instrucción de secundaria así también la mayoría de la población encuestada tiene un estado civil soltero, el mayor número de encuestados son comerciantes, seguidos de los empleados privados, los rangos de edad predominantes son jóvenes de 18 a 27 y adultos de 30 a 35 años, el rango de ingreso que más predominó fue de $\$ 350$ a $\$ 500$, más de la mitad de los encuestados asisten anual y semestralmente a eventos sociales, los eventos de mayor asistencia son bodas y de graduación académica, el $100 \%$ de las personas encuestadas dicen fijarse en la buena comida, seguido de un porcentaje considerable que prefiere la buena atención, la mayor parte de los encuestados prefieren reservar el servicio de catering con un mes de anticipación, en su mayoría, las personas prefieren platos con comida tradicional, a las personas encuestadas les agrada la comida caliente, seguida de la comida al ambiente, más de la mitad de los encuestados dijeron preferir un plato pequeño por persona para el plato de entrada y la población no tiene mayor preferencia por alguna bebida en específico entre gaseosas, agua o vino.

\section{Estrategias}

Penetración rápida, se prevé lanzar al mercado el servicio de catering banquete y buffet con precios bajos en comparación a la competencia, acompañado de grandes costos en promoción, el objetivo que se persigue es una rápida penetración y participación en el mercado, esta estrategia estará en uso hasta que el producto llegue a su etapa de madurez.

Tabla2. Ingenieria de precios

\begin{tabular}{cccrcccc}
\hline \multicolumn{2}{c}{ GAMAS PRODUCTO 1 } & \multicolumn{3}{c}{ GAMAS PRODUCTO 2 } \\
\hline \multirow{2}{*}{ GAMA ALTA } & SUPERIOR & $\$$ & 16,00 & GAMA ALTA & SUPERIOR & $\$$ & 20,00 \\
& INFERIOR & $\$$ & 14,00 & GAMA MEDIA & SUPERIOR & $\$$ & 17,33
\end{tabular}




\begin{tabular}{|c|c|c|c|c|c|c|c|}
\hline \multirow{3}{*}{ GAMA MEDIA } & INFERIOR & $\$$ & 12,00 & & INFERIOR & $\$$ & 14,67 \\
\hline & SUPERIOR & $\$$ & 12,00 & \multirow{2}{*}{ GAMA MEDIA } & SUPERIOR & $\$$ & 1,67 \\
\hline & INFERIOR & $\$$ & 10,00 & & INFERIOR & $\$$ & 12,00 \\
\hline
\end{tabular}

Los productos propuestos se los ha clasificado en tres gamas según el método de Omnes, con un coeficiente de precio ligeramente igual al mínimo de mercado, para cada uno de los productos.

Manual de marca, define los límites y alcances de la marca corporativa, así como su respectiva utilización y usos no permitidos, pues ninguna marca queda completa sin este punto, gracias a esto se logrará posicionar la marca de la asociación en la mente de los consumidores, pues esta hablará por si sola sobre los servicios que se ofrecerán.

\section{Gráfico 1. Marca}

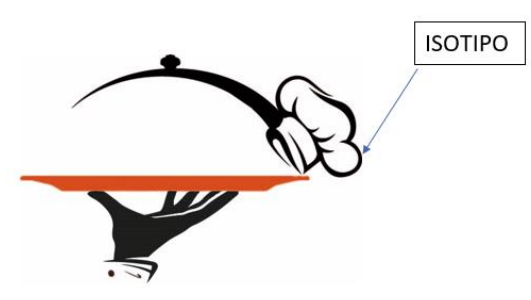

\section{Tipografía} KARA KUNA

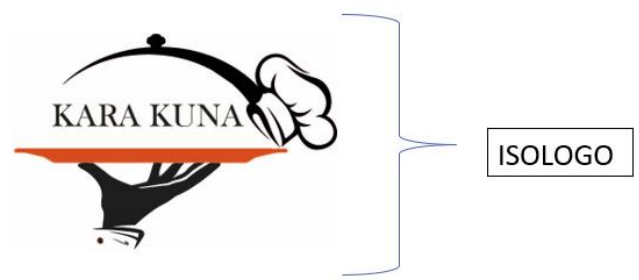

El manual de marca en principio está compuesto por un logotipo, de clasificación Isologo que lo componen, como su nombre lo indica, isotipo y tipografía, la tipografía es de la familia Baskerville y la tipografía la componen una mano, un gorro de chef y un bosquejo de cloche o cubre platos de color negro y un plato de color anaranjado el mismo que se componer según la familia de colores primos, CMYK por $9.23 \%$ de Cían, $80.96 \%$ de magenta, 94.74 de amarillo, y 1.95 de negro. Todas estas figuras se sobreponen según se muestran en las imágenes que anteceden.

\section{Diseño del packaging}

El packaging tiene como objetivo primario atraer la atención de los clientes y ser la principal ventana de comunicación entre el producto y el consumidor, pues es considerado el vendedor silencioso. Este permite obtener una ventaja competitiva para la diferencia de la competencia, además de que transporta, informa y comunica sobre el servicio que se ofrece.

Material: servilletas de papel o fibra, banner hecho a base de lona front.

Características: servilletas blancas con logo negro en el centro, el banner informará de manera sencilla pero llamativa sobre la asociación y sus servicios. 
Propiedades: servilletas con medidas de $20 \mathrm{~cm}$ x $20 \mathrm{~cm}$, banner con medidas de $1,73 \mathrm{~cm}$ x $80 \mathrm{~cm}$.

\section{Gráfico 2. Packaging}
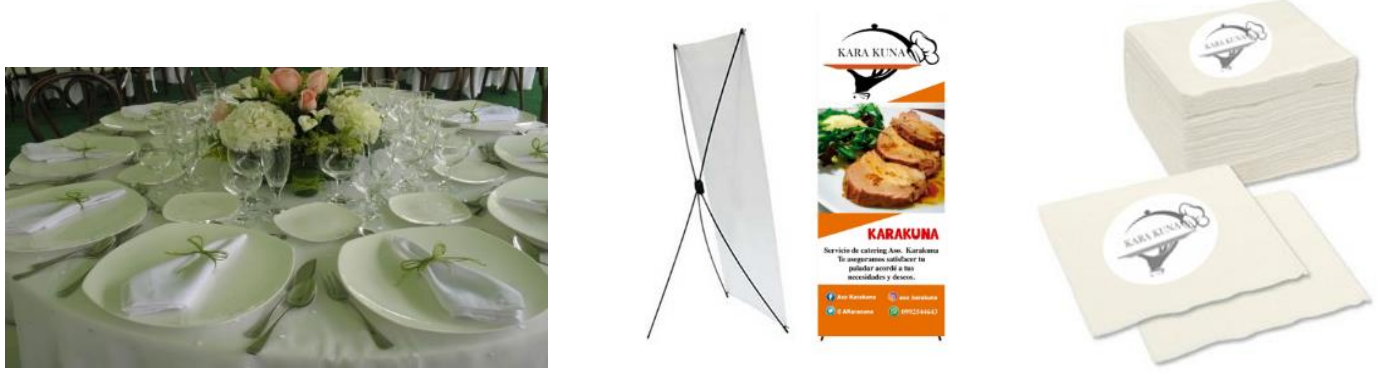

\section{Estrategias de precios}

\section{Descuento en precio por temporadas}

Esta estrategia a corto plazo impacta de forma directa en las ganancias de la asociación. Además, permite cumplir los objetivos de ventas, mejorar la reputación de la empresa y ayuda a que la misma gane más mercado, pues persuade a los clientes actuales y a los potenciales para que creen costumbre de contratar el servicio de catering también en fechas navideñas, informándoles que se realizarán descuentos por la época. Estos descuentos serán del $8 \%$ en fechas navideñas a partir de pedidos para 20 personas.

Tabla 3. Promoción de ventas

\begin{tabular}{|c|c|c|c|c|c|c|c|c|c|c|c|c|c|}
\hline \multicolumn{2}{|c|}{$\begin{array}{c}\text { PRECIO DE } \\
\text { VENTA } \\
\text { BANQUET } \\
\text { E } \\
\end{array}$} & \multirow[t]{2}{*}{$\begin{array}{c}\text { \# DE } \\
\text { MENUS } \\
\text { VENDIDO } \\
\text { S } \\
\end{array}$} & \multicolumn{2}{|c|}{$\begin{array}{c}\text { COSTO DE } \\
\text { FABRICACIÓ } \\
\mathbf{N}\end{array}$} & \multicolumn{2}{|c|}{$\begin{array}{c}\text { TOTAL DE } \\
\text { COSTOS DE } \\
\text { FABRICACIÓ } \\
\mathbf{N}\end{array}$} & \multicolumn{2}{|c|}{$\begin{array}{c}\text { PRECIO DE } \\
\text { VENTA SIN } \\
\text { DESCUENTO }\end{array}$} & \multicolumn{2}{|c|}{$\begin{array}{l}\text { DESCUENT } \\
\text { O 8\% }\end{array}$} & \multicolumn{2}{|c|}{$\begin{array}{c}\text { PRECIO } \\
\text { VENTA CON } \\
\text { DESCUENT } \\
\text { O }\end{array}$} & $\begin{array}{c}\text { GANACIA } \\
\mathbf{S} \\
\text { LÍQUIDAS }\end{array}$ \\
\hline$\$$ & 16,00 & & $\$$ & 11,00 & $\$$ & 220,00 & & 20,00 & & 25,60 & & 294,40 & 4,40 \\
\hline$\$$ & 16,00 & 30 & $\$$ & 11,00 & $\$$ & 330,00 & & 80,00 & $\$$ & 38,40 & & 441,60 & $\$ 11,60$ \\
\hline$\$$ & 16,00 & 40 & $\$$ & 11,00 & $\$$ & 40,00 & & 640,00 & $\$$ & 1,20 & & 588,80 & $\$ 148,80$ \\
\hline \multicolumn{2}{|c|}{$\begin{array}{c}\text { PRECIO } \\
\text { DE VENTA } \\
\text { BUFFET }\end{array}$} & $\begin{array}{c}\text { \# DE } \\
\text { MENUS } \\
\text { VENDIDO } \\
\mathbf{S}\end{array}$ & \multicolumn{2}{|c|}{$\begin{array}{l}\text { COSTO DE } \\
\text { FABRICACIÓ } \\
\mathbf{N}\end{array}$} & \multicolumn{2}{|c|}{$\begin{array}{c}\text { TOTAL DE } \\
\text { COSTOS DE } \\
\text { FABRICACIÓ } \\
\mathbf{N}\end{array}$} & \multicolumn{2}{|c|}{$\begin{array}{c}\text { PRECIO DE } \\
\text { VENTA SIN } \\
\text { DESCUENTO }\end{array}$} & \multicolumn{2}{|c|}{$\begin{array}{c}\text { DESCUENT } \\
\text { O 8\% }\end{array}$} & \multicolumn{2}{|c|}{$\begin{array}{c}\text { PRECIO } \\
\text { VENTA CON } \\
\text { DESCUENT } \\
\text { O }\end{array}$} & $\begin{array}{l}\text { GANACIA } \\
\mathbf{S} \\
\text { LÍQUIDAS }\end{array}$ \\
\hline$\$$ & 20,00 & 20 & $\$$ & 16,00 & $\$$ & 320,00 & $\$$ & 400,00 & & $\begin{array}{r}\$ \\
32,00 \\
\$\end{array}$ & $\$$ & 368,00 & $\begin{array}{r}\$ \\
48,00 \\
\$\end{array}$ \\
\hline$\$$ & 20,00 & 30 & $\$$ & 16,00 & $\$$ & 480,00 & $\$$ & 600,00 & & $\begin{array}{r}48,00 \\
\$\end{array}$ & $\$$ & 552,00 & $\begin{array}{r}72,00 \\
\$\end{array}$ \\
\hline$\$$ & 20,00 & 40 & $\$$ & 16,00 & $\$$ & 640,00 & $\$$ & 800,00 & & 64,00 & $\$$ & 736,00 & 96,00 \\
\hline
\end{tabular}

Fuente: Elaboración propia

En la tabla se puede observar el precio de venta con descuento del servicio de catering banquete y buffet, así como la rentabilidad que se obtendrá por las diferentes cantidades vendidas. 


\section{Descuento por cantidad}

El descuento será una reducción en el precio del servicio que prestará Aso Kara Kuna, aquí gana el cliente, pero también los socios de la asociación. Esta estrategia permitirá aumentar el volumen de ventas por platos para cada evento, la disminución del precio total se dará por el contrato del servicio para 50 o más personas.

El costo de fabricación del producto, así como la venta con descuento del mismo y su respectiva ganancia liquida según la cantidad demandada, el descuento asciende mientras más sean los platos pedidos.

\section{Estrategias de comunicación}

\section{Diseño de la carta}

Es importante contar con menús pre establecidos, pues la carta es un medio de comunicación sin igual que habla por sí sólo. Permitir que los clientes se informen de la variedad de productos que ofrecerá la asociación, según sus diferentes necesidades, gustos y preferencias. Plasmar en cartas los diferentes menús que la empresa ofrecerá

Material: cartas impresas en papel couche.

Características: diseño colorido, elegante y llamativo, con descripción del menú e imagen del plato fuerte en la parte superior.

Propiedades: medidas $21 \mathrm{~cm}$ x $29,7 \mathrm{~cm}$

Gráfico 3. Diseño de la nueva carta
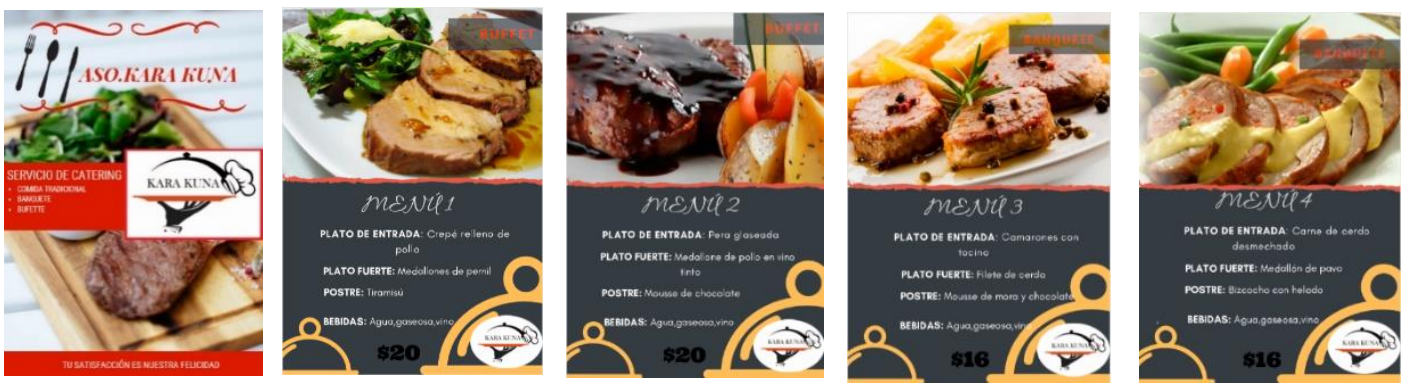

\section{Uniformes}

El uniforme no es sólo una parte distintiva de la empresa o de los clientes internos de la misma, pues este también ayuda a generar confianza al cliente externo porque transmite una imagen de seriedad y respeto. Esta estrategia busca generar presentación y buena atención del servicio, a través del correcto uso del uniforme.

Material: Uniforme a base de tela de algodón

Características: tela color negro sable, combinado con blanco y rojo, además del logotipo de la asociación.

Propiedades: Tallas s, m, x, 1 
Gráfico 4. Uniformes
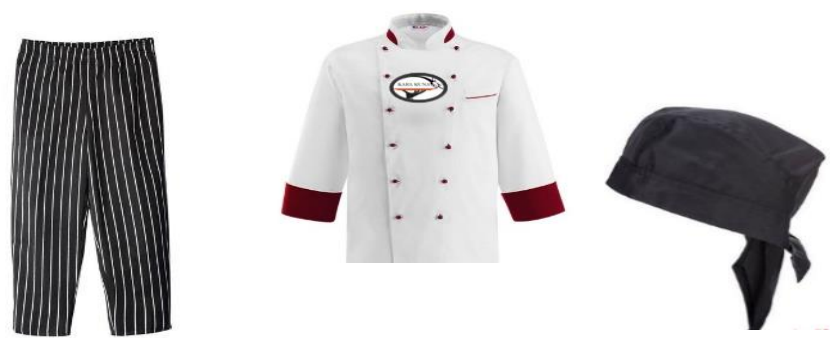

\section{Comunicación en Facebook}

Facebook es una red social, es un espacio que permite compartir contenido de forma sencilla a través de Internet. Aquí los usuarios pueden mantenerse en contacto continuo con la asociación y los servicios que se prestarán en el mercado riobambeño, las publicaciones serán las 24 horas, los siete días de la semana y el contenido será sobre platos elaborados.

\section{Gráfico 5. Facebook}

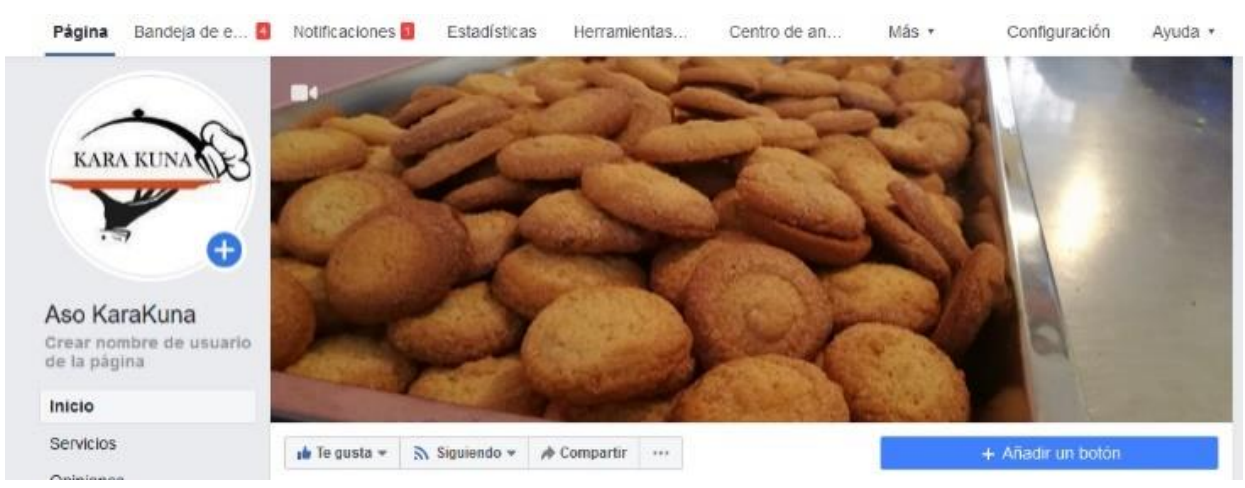

\section{Comunicación en Instagram}

Instagram es una red social y una aplicación móvil al mismo tiempo. Esta permite a sus usuarios subir imágenes y vídeos con múltiples efectos fotográficos, es por eso que ayudará a generar reconocimiento de la asociación de forma creativa, pues las publicaciones serán las 24 horas del día, todas las semanas, haciendo énfasis en la salud alimenticia y las propiedades de los alimentos.

\section{Gráfico 6. Instagram}

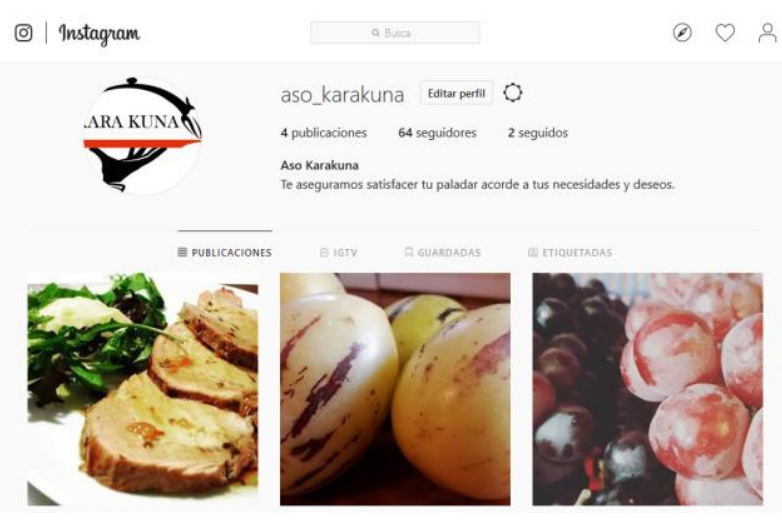




\section{Below the line (Btl)}

Consiste en emplear formas de comunicación no masivas dirigidas a un segmento específico, empleando como principales recursos la creatividad, la sorpresa y el sentido de oportunidad, estas estrategias buscan crear mensajes novedosos para comunicar los servicios de Aso Kara Kuna.

Alternativa 1 - Árbol con tallo de tenedor

Material: Resistente al agua, acero inoxidable

Características: Tenedor color plomo con el logo e información de la asociación.

Propiedades: 1, $50 \mathrm{~m}$ de diámetro por $5 \mathrm{~m}$ altura.

Gráfico 7. Estrategia Btl

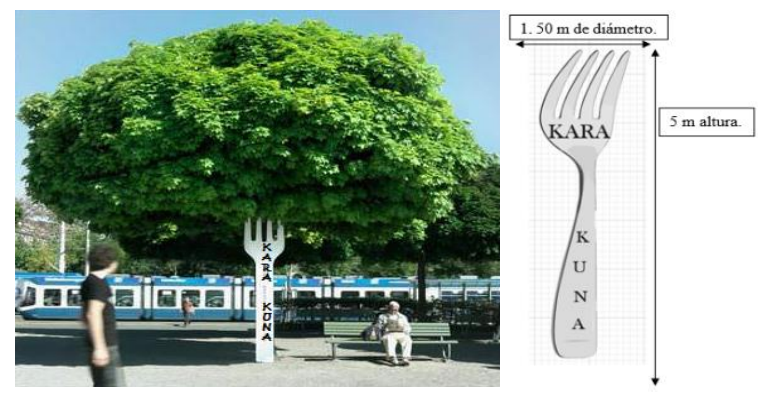

Alternativa 2- Asiento público con forma de tabla de cocina.

Material: Pino fresco

Características: asiento con respaldar llamativo, el cual llevará el logo de la asociación Propiedades: $1,16 \mathrm{~m}$ por $75 \mathrm{~cm}$

Gráfico 8. Estrategia Btl
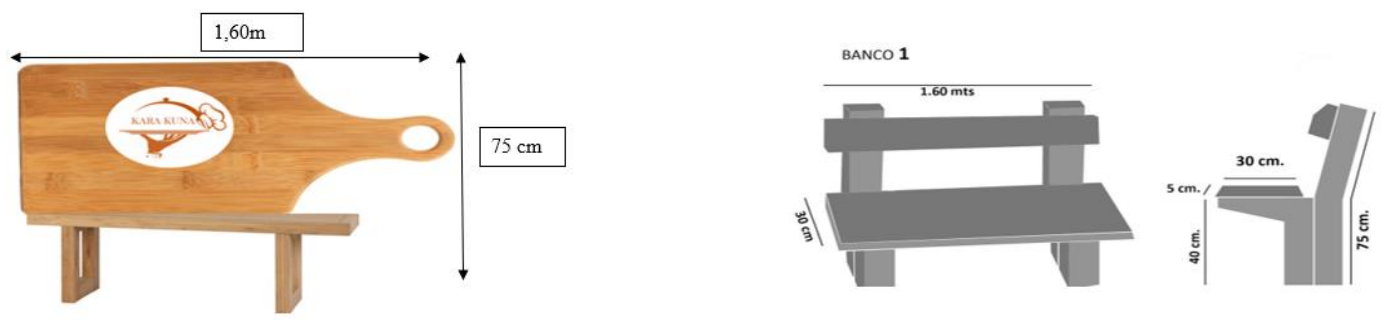

Alternativa 3- Paso cebra con cubiertos de mesa

Material: Pintura vinílica

Características: paso cebra llamativa, con imágenes que representen al servicio de Kara Kuna

Propiedades: $60 \mathrm{~cm}$ por $1,80 \mathrm{~m}$ 
Gráfico 9. Estrategia Btl
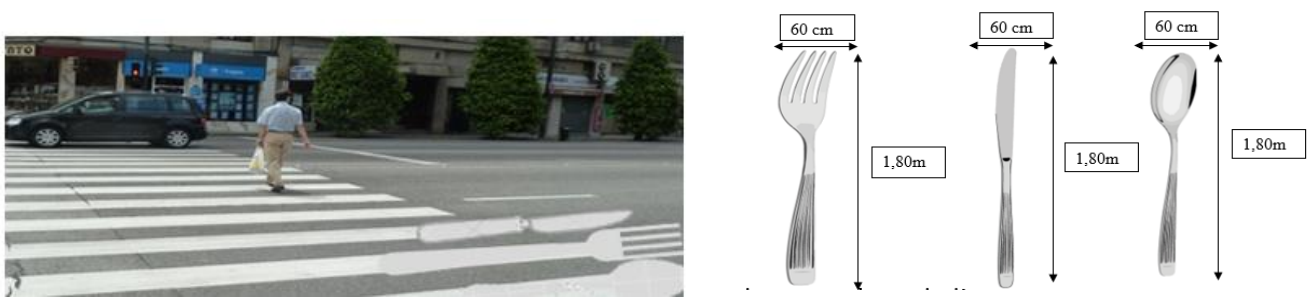

Alternativa 4- Gorro de chef

Material: tela impermeable

Características: gorro gigante color blanco, con el logo de la asociación

Propiedades: $1 \mathrm{~m}$ por $1,60 \mathrm{~m}$

Gráfico10. Estrategia Btl
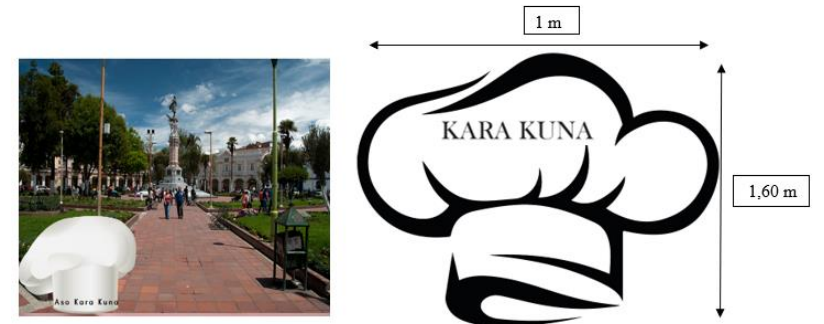

Alternativa 5- Stickers publicitarios

Material: vinilo

Características: pegatinas en forma de pie color negro que se dirigen a un plato creativo Propiedades: plato de $1 \mathrm{~m}$ por 1,50 y pies de $30,48 \mathrm{~cm}$ por $15 \mathrm{~cm}$

Gráfico 11. Estrategia Btl
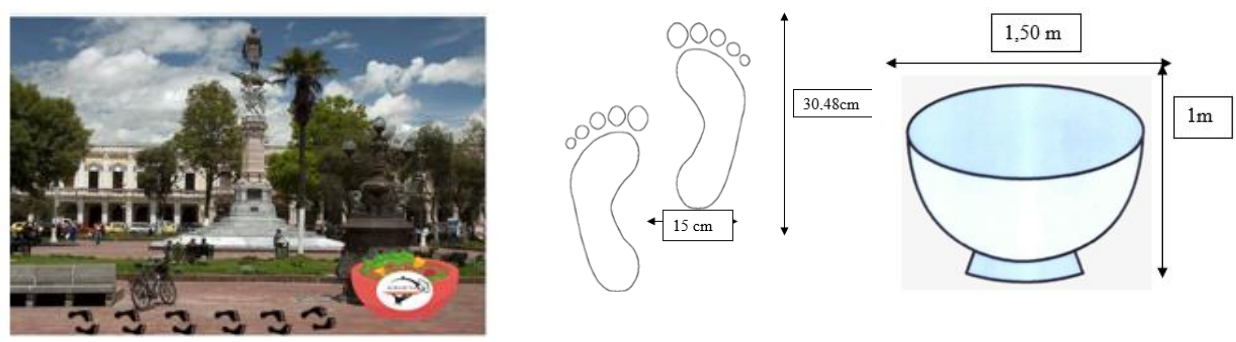

Aso kara kuna brinda productos de la Economía Popular y Solidaria y estos son considerados de pobres para pobres por lo cual a través de este tipo de investigaciones se rompe con este paradigma. Las métricas fueron desarrolladas para la población en general por esta razón se sostiene que los productos de la Economía Popular y Solidaria son para cubrir las necesidades de la población ecuatoriana sin identificar segmentos socioeconómicos puntuales; pues estos productos son considerados de calidad porque son elaborados con estándares de normalización y sanidad correspondientes.

Es por esto que para el desarrollo del nuevo servicio de cáterin para Aso-Kara Kuna se destaca que los clientes al ser comerciantes tienen la capacidad adquisitiva para solicitar 
el servicio de alimentación, además más de la mitad de los encuestados asisten anual y semestralmente a eventos sociales tales como de bodas y graduaciones, dado que el cáterin es indispensables para el correcto desarrollo de las reuniones y festejos en los cuales la familia y los amigos se juntan y se liberan de la responsabilidad de servir los alimentos a los invitados, en definitiva para Aso Kara Kuna la investigación y desarrollo del producto es la parte del marketing, necesaria para que el servicio tenga éxito en el mercado.

\section{Conclusiones.}

- Aso Kara Kuna es una asociación comprometida con su trabajo, tiene muchas fortalezas para seguir creciendo empresarialmente, una vez analizada la situación interna y externa de la misma, se deduce que la asociación está dentro de las mejores en su giro de negocio.

- Los productos de la economía popular y solidaria son productos que cuenta con los estándares de calidad para el consumo de la población, ya que la economía popular y solidaria fue creada para mejorar las condiciones de vida de la sociedad.

- Mediante la aplicación de la investigación de mercados en la zona urbana de la ciudad de Riobamba debido se obtuvo el panorama real del mercado en el cual aso Kara Kuna desea prestar el servicio de Catering.

- En los resultados de la investigación de mercados se obtuvo dos perfiles de consumidor para la asociación, el primero comprende a personas solteras de 18 a 24 años, las cuales asisten a eventos de forma mensual y el otro de 30 a 35 años, con un estado civil casado, es decir familias jóvenes, este fenómeno hace que asistan de forma semestral a eventos sociales y busquen alimentos más saludables.

- Aso Kara Kuna tienen gran aceptación en el mercado Riobambeño para el servicio de catering de banquete y buffet, pues las personas manifestaron que les agradaría mucho contratar dicho servicio.

- Las estrategias propuestas para cumplir los objetivos organizacionales van acordes con la capacidad adquisitiva de la asociación y al giro del negocio actual, estas son económicas y su aplicación traerán grandes beneficios.

\section{Referencias bibliográficas.}

Canales, Pedro. (2013). "Neuromarketing: Marketing, throught Science. IEEE Computer Society, págs. 285-289.

Cravens, David \& Piercy, Nigel. (2007) Marketing Estrategico. Madrid : Mc Graw-Hill, pág. 29.

Garnica, Glotilde., Maubert, Viveros \& Claudio, Alfonso. (2009). Fundamentos del marketing. Mexico : Pearson, pág. 18.

Gómez, Claudia., \& Mejía, Eduardo. (2012). La gestión del marketing que conecta con los sentidos, Revista Escuela de Administración de negocios , pág. 7. 
Hoffman, Czinkota., Dickson, Dunne., \& Griffin, Hutt. (2007). Principios del marketing y sus mejores practicas. Principios del marketing y sus mejores practicas. Mexico : THOMSON, pág. 10.

Kotler, Philip., Keller, Kevin. (2016). Direccion de marketing. Mexico: Pearson Educacion, pág. 7.

Mesa, Mario. (2012). Fundamentos del marketing. Bogota : Eco Ediciones, pág. 99.

Lambin, Jean. (1995). Marketing estratégico. Paris : McGRAW-HILL, pág. 8.

Ley Orgánica de la Economía Popular y Solidaria. (2011). Ley EPS del sector financiero popular y solidario. Quito : Registro Oficial, Vol. 2, 3.

Linton, Ian. (2019). La voz de Houston . Como crear un proceso de investigación y desarrollo. [En línea] https://pyme.lavoztx.com/cmo-crear-un-proceso-deinvestigacin-y-desarrollo-8920.html.

Pacheco, Iván. (2018). Superintendencia de Economa Popular y Solidaria. Conoce que es la Economía Popular y Solidaria (EPS). [En línea] http://www.seps.gob.ec/noticia?conoce-la-eps.

Pérez, David. (2018). Marketing para Abogados escépticos. Ebook central proquest. [En línea]. http://ebookcentral.proquest.com/lib/espochsp/detail.action?docID=5513806.

Sánchez, Joaquin. (2010). Estrategias y planificación en marketing . Estrategias y planificación en marketing . Madrid : Anaya,S.A.

SENPLADES. (2013). Plan Nacional del Buen Vivir . Plan Nacional del Buen Vivir. Gobierno Ecuatoriano www.buenvivir.gob.ec. Quito: Registro Oficial.

Vallet, Teresa. (2015). Principios de marketing Estrategico. Castellon : I-UNE, pág. 9.

Zabala, Harold. (2017). Neoromarketingcomo apoyo al merchandising en las tiendas. Eumednet, pág. 17.

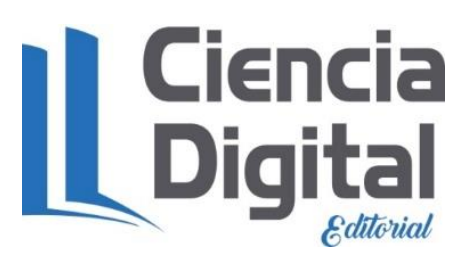

PARA CITAR EL ARTÍCULO INDEXADO. 
Naranjo Silva, E. R., Zabala Jarrín, H. A., \& Vistin Guamantaqui, D. A. (2021). Investigación y desarrollo de un nuevo producto para emprendimiento de microeconomía $\begin{array}{lllll}\text { popular } & \text { y } & \text { solidaria. } & \text { ConcienciaDigital, } & 4(3.1),\end{array}$ https://doi.org/10.33262/concienciadigital.v4i3.1.1819

\section{LCiencia}

El artículo que se publica es de exclusiva responsabilidad de los autores y no necesariamente reflejan el pensamiento de la Revista Conciencia Digital.

El artículo queda en propiedad de la revista y, por tanto, su publicación parcial y/o total en otro medio tiene que ser autorizado por el director de la Revista Conciencia Digital.

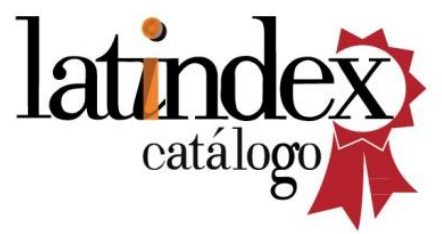

